\title{
A Cost-effective TEQ Algorithm for ADSL Systems
}

\author{
Chih-Chi Wang and An-YeuWu \\ Department of Electrical Engineering, \\ National Taiwan University, \\ Taipei 106, Taiwan, R.O.C
}

\begin{abstract}
Discrete multitone (DMT) modulation/demodulation scheme is the physical-layer standard of Asymmetric digital subscriber line (ADSL) system. In DMT transceiver, channel equalization is completed through two steps, namely, Time-domain Equalization (TEQ) and Frequency-domain Equalization ( $F E Q$ ). The TEQ is introduced to shorten the channel response to a pre-defined length. On the contrary, FEQ is used to compensate for the magnitude and phase distortion caused by channel. In this paper, a cost-effective on-line TEQ training algorithm is proposed. Simulation results show that the proposed TEQ algorithm has comparable performance with other existing on-line algorithms, and it achieves the lowest computational complexity among these TEQ algorithıns.
\end{abstract}

\section{Introduction}

DMT transmission technology is a form of multicarrier modulation. In this modulation scheme, the communication channel is divided into a bank of orthogonal subchannels in frequency domain. In DMT technology, every symbol consists of $N$ sample that is prefixed by its last $v$ samples, called cyclic prefix $(C P)$, to eliminate the inter-symbol interference (ISI). The $\mathrm{CP}$ is redundant for current symbol and makes bit rate decreased by the factor of $v /(N+v)$. To maintain a higher data rate, a small $v$, or equivalently, a short channel response is desired. Unfortunately, the length of the channel response for ADSL system is usually very long, so that $v$ is generally a large number. In order to obtain a small $v$, Shortened Impulse Response Filter $(S I R F)$ [1], or so-called Time-domain Equalizer $(T E Q)$ [2], is added in the receiver to shorten the length of the channel response, as shown in Fig. 1.

Frequency-domain LMS (FLMS) and Time-domain LMS (TLMS) can be viewed as on-line algorithm since they utilize the Least Mean-Squares (LMS) algorithm to estimate the TEQ coefficients as data arrive. Generally, FLMS and TLMS achieve lower complexity than off-line TEQ training algorithms that utilize the matrix computation [3]. However, the complexity of FLMS and TLMS is still high from the viewpoint of practical implementations. In this paper, we derive a novel on-line TEQ training algorithm, called Time-and-Frequency domain TEQ (TF-TEQ), which can further lower the hardware/computational complexity of TEQ algorithm. Base on the periodicity of training sequence, we try to train the TEQ coefficients in the mixed frequency and time domains. By doing so, the proposed algorithm can save about $45 \%$ computational complexity than conventional FLMS and TLMS algorithms.

\author{
Bor-Min Wang \\ N200 CCL/ITRI 195-1I Sec.4 \\ Chung Hsing Rd., Chutung, \\ Hsinchu 310, Taiwan, R.O.C.
}

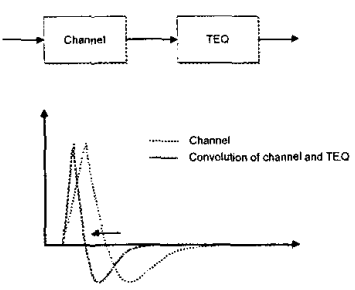

Fig. 1 Time-domain equalizer (TEQ) in DMT transceiver.

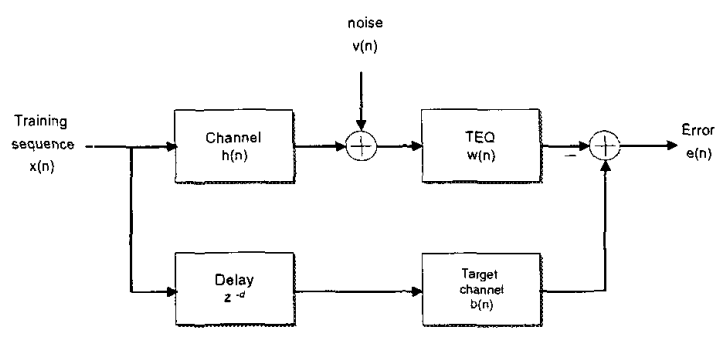

Fig. 2 Block diagram of the TEQ problem.

\section{Time Domain Equalization in DMT Transceiver}

TEQ problem can be modeled as the block diagram shown in Fig. 2. Let $h(n)$ and $w(n)$ represent the coefficients of channel and the response of TEQ respectively. Target channel $b(n)$ is an arbitrary FIR filter which has only $v+1$ taps. The goal of TEQ is to equalize the channel so that the cascade of $h(n)$ and $w(n)$ equals to $b(n)$, i.e.,

$h(n) * u(n)==\delta(n-d) * b(n)$,

where the parameter, $d$, denotes the channel delay. There are many on-line algorithms have been proposed in literature to train TEQ for ADSL systems. Based on the periodicity of training sequence, Chow et al. [4] proposed an LMS-based algorithm, which operates in the frequency domain, to train the TEQ coefficients. The algorithm is referred as Frequency domain LMS (FLMS). In FLMS algorithm, target channel and TEQ are updated simultaneously in the frequency domain for each DMT training symbol. Fig. 3 shows the block diagram of FLMS algorithm. Let $X(k)$ and $Y(k)$, for $k=0$ to $N-1$, represent the FFT of the received symbol, $y(n)$, and training symbol, $x(n)$, respectively. $W_{i}(k)$ and $B_{i}(k)$ are the FFT of $w(n)$ and $b(n)$. The frequency domain coefficients, $W_{i}(k)$ and $B_{i}(k)$, with subscription $i$ denotes the $i$ th updated result. For each symbol, the algorithm performs the following procedures: 
$E(k)=B_{i}(k) X(k)-W_{i}(k) Y(k)$

$B_{i+1}(k)=B_{i}(k)+\mu_{b} E(k) X^{*}(k)$

$W_{i+1}(k)=W_{i}(k)+\mu_{w} E(k) Y^{*}(k)$

For $k=0,1, . ., N-1$

in frequency domain. $B(k)$ and $W(k)$ are transformed back to time domain and performed a window on them for each update. Basically, the principle updating process of FLMS is "updating in frequency domain and windowing in time domain." To operate in frequency domain, the FLMS algorithm needs several FFT/IFFT transforms to perform the transformation of coefficients between time and frequency domains. Hence, the complexity of FLMS is still high.

Based on the similar idea of FLMS algorithm, we can update $b(n)$ and $w(n)$ simultaneously in the time domain. In contrary to FLMS algorithm that operates in the frequency domain, this approach is called Time-domain LMS (TLMS) [5] algorithm. Considering the adaptive filter problem in Fig. 4, TEQ and the target channel are simply $L$-tap and $(v+1)$-tap FIR filters, respectively. The TLMS utilizes the LMS algorithm to update the coefficients of $w(n)$ and $b(n)$ simultaneously. At the beginning of TEQ training, the coefficients of $w(n)$ and $b(n)$ are set to some initial values, and the following procedures are performed on each incoming sample:

$$
\begin{aligned}
& e(n)=\mathbf{b}_{n}^{T} \mathbf{x}_{n}-\mathbf{w}_{n}^{T} \mathbf{y}_{n} \\
& \mathbf{b}_{n+1}=\mathbf{b}_{n}-\mu_{b} e(n) \mathbf{x}_{n} \\
& \mathbf{w}_{n+1}=\mathbf{w}_{n}+\mu_{w} e(n) \mathbf{y}_{n}
\end{aligned}
$$

If the step size, $\mu_{b}$ and $\mu_{w}$ and the initial value of $w(n)$ and $b(n)$ are chosen properly, the coefficients of TEQ and target channel will converge to the target solution. In general, TLMS converges faster than FLMS. However, the updating procedures are operated for every sample, so that the complexity is vary high, especial for a long-tap TEQ. The detailed operations can be found in [4] and [5].

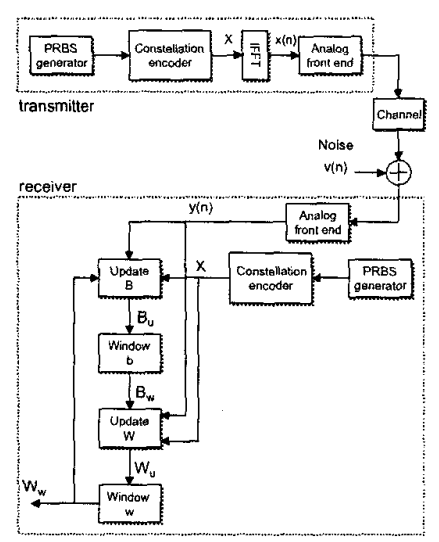

Fig. 3 Frequency-domain LMS algorithm in [4].

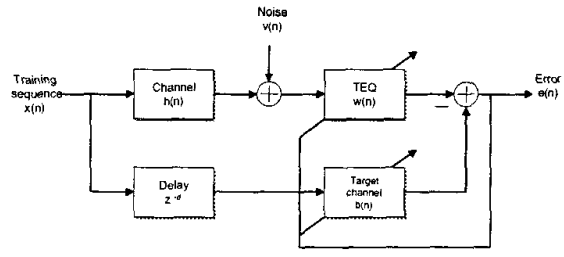

Fig. 4 Block diagram of Time-domain LMS.

\section{The Proposed TF-TEQ algorithm}

\section{A. Basic idea}

The block diagram of the proposed algorithm is shown in Fig. 5 . During the initialization of DMT transceiver, the transmitter sends repeated training symbols over channel. The repeated symbols form periodic signal and perform circular convolution with channel, $h(n)$. Assume that the training symbols sent to channel is $x(n)$, for $n=0$ to $N-1$. We can consider the received symbol, $u(n)$, as

$u(n)=h(n) \otimes x(n)+v(n)$,

where $v(n)$ denotes the additive white noise and $\otimes$ denotes the operation of circular convolution. At receiver end, we utilize the adaptive filtering algorithm to train TEQ according to the known training sequence. To speed up the convergence rate, we introduce a function block, called channel estimator, to perform the immediate estimation of effective channel,

$h_{\text {eff }}(n)=h(n) \otimes w(n)$

for each update. $w(n)$ represents the coefficients of TEQ, and $h_{e s t}(n)$ represents the immediate estimation of $h_{\text {eff }}(n)$ done by channel estimator. We set the coefficients of target channel to $h_{e s t}(n)$, then apply the LMS algorithm to update TEQ coefficients according to the updated coefficient, $h_{e s s}(n)$. If $h_{e s t}(n)$ can estimate the effective channel approximately, the difference between $h_{\text {eff }}(n)$ and $h_{\text {est }}(n)$ is small. Hence, the rate of convergence can be speeded up.

\section{B. Derivation of Algorithm}

We can write the equalized signal, $y(n)$, as

$y(n)=h_{e f f}(n) \otimes x(n)+v(n)$

Taking FFT on both sides, Equation (6) becomes

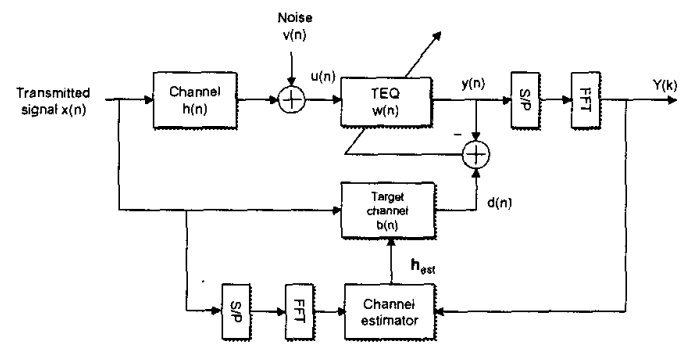

Fig. 5 Block diagram of our TF-TEQ algorithm. 
$Y(k) \cong H_{e f f}(k) X(k)$

Here, we can ignore the effect of noise because it becomes negligible when we average the observation of $Y(k)$ over a long period of time. We can estimate the effective channel by applying division property, i.e.,

$H_{\text {eff }}(k)=Y(k) / X(k)$

The estimation of effective channel done by (5) should be in the situation that $w(n)$ is fixed. However, when we utilize the adaptive filtering algorithm to train TEQ, $w(n)$ is varied with time. Hence, we divide the progress of training into two phases, called Channel-estimating phase and Channel-updating phase, as shown in Fig. 6. Each phase lasts for one DMT symbol time. At channel-estimating phase, the coefficients of TEQ are fixed until one whole received DMT symbol (equivalently, 512 samples for ADSL standard) is filtered. At the end of channel updating phase, channel estimator calculates the estimation of $h_{e f f}(n)$ by (8). Then, the TEQ enters the channel-updating phase. At channel updating phase, TEQ is updated using the LMS algorithm on each sample for 512 times. Then we go back to the channel-estimating phase.

Fig. 7 shows the detailed operations of our channel estimator The divider performs the division operation of $Y$ and $X$ element-by-element. The estimation of effective channel in time domain is then calculated by taking IFFT on the divided result. A rectangular windowing function is performed to limit the estimated result to $v+1$ samples. Here, rectangular windowing function is chosen because it can be easily realized by retaining the samples inside the window and forces the others to zero. Besides, we need to normalize the energy of $h_{\text {est }}(n)$ to a preset value to avoid the trivial solution of $w(n)=h_{\text {est }}(n)=\left[\begin{array}{lll}0 & 0 & \ldots\end{array}\right]$.

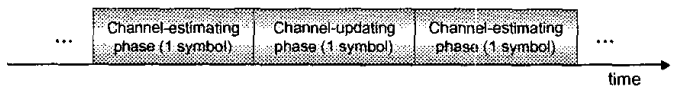

Fig. 6 Channel-estimating phase and channel updating phase.

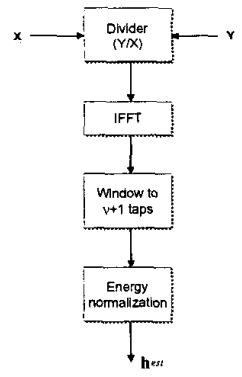

Fig.7 Block diagram of channel estimator.
The operations related to (8) involve the division. In practice, we do not need to realize a real divider to perform the division operation. Because $X(k)$ is known in advance during TEQ training, and we can pre-store the values of $1 / X(k)$ in memory. The division of $Y(k)$ and $X(k)$ now becomes the multiplication of $Y(k)$ and $1 / X(k)$. We can easily perform the division operation without using a real divider in practical implementation.

In the channel-updating phase, the TEQ is updated sample-by-sample. TEQ is simply an $L$-tap adaptive FIR filter. Assume that the output of target channel in the channel-updating phase is $d(n)$ for $n=0,1, \ldots$ to 511 , and the vector

$\mathbf{u}(n)=[u(n) u(n-1) \ldots u(n-L+2) u(n-L+1)]^{T}$

represents the tap-input vector of TEQ at time $n$. We can update the weights of the $L$-tap FIR by using LMS algorithm. That is,

$e(n)=d(n)-\mathbf{w}^{T}(n) \mathbf{u}(n)$,

$\mathbf{w}(n+1)=\mathbf{w}(n)+\mu_{w} \mathbf{u}(n) e^{*}(n)$.

where $\mathbf{w}(n)=\left[\begin{array}{llll}w_{0}(n) & w_{1}(n) \quad \ldots w_{L-1}(n)\end{array}\right]^{T}$ denotes the tap-weight vector of TEQ at time $n$. The TEQ will be updated for 512 times, and then enter the channel-estimating phase.

\section{The Delayed TF-TEQ Algorithm}

At the end of channel-estimating phase in the proposed algorithm, the channel estimator performs the channel estimation and the windowing procedures. The estimated result, $h_{e s}(n)$, is used right away for the subsequent channel-updating phase. The time for the operations of channel estimator is only one sample. It is almost impossible to achieve real-time processing. Hence, we modify our TF-TEQ algorithm to a delayed version as shown in Fig. 8. The idea is similar to the Delayed LMS (DLMS) algorithm [6]. The current coefficients of target channel are set to $h_{e s t, d}(n)$, the last estimation of effective channel, where the subscript $d$ represents the coefficients are delayed. The current estimation, $h_{e s t}(n)$, is delayed for two symbols. Consequently, the time for the operations of channel estimator is extended to two whole DMT symbols (because the effective channel is estimated for every two symbol). This eases the design of channel estimator and makes our algorithm more suitable for practical implementations. Simulation results in Section V also show that the modified algorithm can work properly.

We summarize the delayed TF-TEQ algorithm in Table 1. Of course, at the beginning of the training phase, parameter $\mathbf{w}, \mathbf{b}$, and step size, $\mu_{m}$, should be set to the proper initial values. 


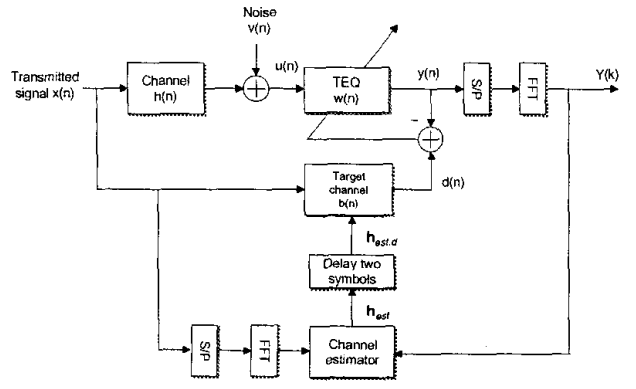

Fig. 8 The delayed TF-TEQ algorithm.

Table 1 Summary of the proposed TF-TEQ algorithm.

\begin{tabular}{|c|c|}
\hline Step 1 & $\begin{array}{l}\text { Estimate the channel, } h(n) \text {, by }(8) \text { while setting } w(n)=[1 \\
00 \ldots 0] \text { and calculate the vergy of } h(n), E_{c h} \text {. }\end{array}$ \\
\hline Step 2 & Update TEQ by using the LMS algorithm in (9). \\
\hline Step 3 & $\begin{array}{l}\mathbf{b}=\mathbf{b} \text { _tmp, where } \mathbf{b} \text { tmp is a temporary vector for sav- } \\
\text { ing the updated result of } h_{\text {est }}(n) \text {. }\end{array}$ \\
\hline Step 4 & Fix $w(n)$ and feed a whole DMT symbol into TEQ. \\
\hline Step 5 & $\begin{array}{l}\text { Collect the demodulated symbol, } Y(k) \text {, and then calculate } \\
h_{\text {est }}(n) \text { according to the design flow in Fig. } 7 \text {. }\end{array}$ \\
\hline Step 6 & b_tmp $=\mathbf{h}_{\text {est }}$, where $\mathbf{h}_{\text {est }}$ is the matrix form of $h_{e s t}(n)$. \\
\hline Step 7 & Go back to step 2 until the design criteria are met. \\
\hline
\end{tabular}

Table 2 summarizes the number of multiplication that the proposed TF-TEQ algorithm requires. Because the number of multiplication of the TF-TEQ algorithm is evaluated for two training symbols (due to the channel-updating phase and channel-estimating phase), the number of multiplication for each training symbol is halved. We summarize the number of multiplication per symbol of the three TEQ algorithms in Fig. 9. From this figure, we can easily inspect the numerical relationship of computational complexity between the three algorithms. We conclude that the FLMS has the highest complexity while our TF-TEQ requires the least complexity.

Table 2 Computational complexity of our TF-TEQ algorithm $(L=16, N=512, v=32)$

\begin{tabular}{|c|c|}
\hline Operation & Num. of multiplication \\
\hline$y=u * w($ channel-updating phase) & $L N$ real \\
\hline$w=w+\mu e^{*} u($ channel-updating phase $)$ & $L N$ real \\
\hline$d=x * \tilde{h}_{e f f} \quad$ (channel-updating phase) & $(v+1) \mathrm{N}$ real \\
\hline$y=u * w$ (channel-estimating phase) & $L N$ real \\
\hline$y \rightarrow Y$ (channel-estimating phase) & $\frac{N}{2} \log _{2} N$ complex \\
\hline$H_{e s t}=\frac{Y}{X}$ (channel-estimating phase) & $N$ complex \\
\hline$H_{e s t} \stackrel{I F F T}{\rightarrow} h_{e s t}$ (channel-estimating phase) & $\frac{N}{2} \log _{2} N$ complex \\
\hline Total (per two symbols) & $60,416 \mathrm{real} / \mathrm{per}$ two symbols \\
\hline Average & $30,208 \mathrm{real} / \mathrm{symbol}$ \\
\hline
\end{tabular}

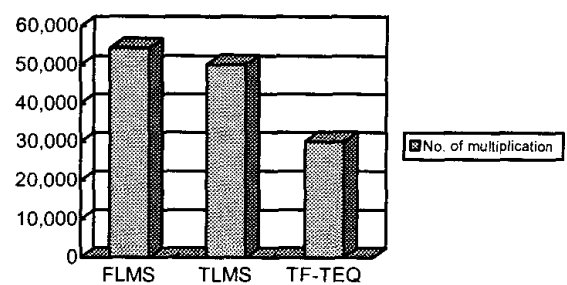

Fig. 9 Comparison of computational complexity.

\section{Simulation Results}

In this section, we conduct several computer simulations to show the shortening performance of our algorithm. All simulations focus on the receiver training at remote site. The FFT size is 512 and the length of CP, $v$, is 32 samples as defined in ADSL standard. During the transmission, the channel will be injected with several noise sources, including Additive white Gaussian noise ( $A W G N$ ), crosstalk noise, etc. Here, we concern only the Far-end crosstalk (FEXT), Near-end crosstalk (NEXT), and AWGN noise. The simulation environment setup is shown in Fig. 10. The following simulation results are conducted based on this environment.

Due to the finite number of tap, TEQ generally cannot perform the perfect shortening of channel. Some energy will lie outside the largest $(v+1)$ contiguous samples of the effective channel, $h_{\text {eff }}(n)$, which is the cascade of channel response and TEQ. To measure the performance of the result, a performance index, called Shortening SNR (SSNR), is defined. Assume that the energy of a window of $(v+$ 1) samples with highest energy is $E_{\text {in }}$, and the energy of samples outside the window is $E_{\text {leakage }}$. Then the $S S N R$ is defined as

$S S N R=10 \log _{10}\left\{\frac{E_{\text {in }}}{E_{\text {leakage }}}\right\}$

The larger the SSNR, the better the shortened performance is achieved. To verify the validity of our TF-TEQ algorithm, we first present the shortened result of our algorithm.

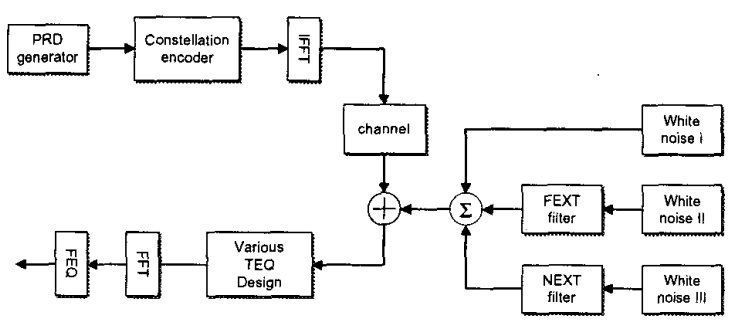

Fig. 10 Simulation environment. 
Table 3 shows the SSNR value of eight testing channels after 500 training symbols are sent. The results of FLMS, TLMS, and TF-TEQ algorithm are listed together for comparison. From the table, we find that the TLMS can achieve good performance for different loops. FLMS sometimes can achieve the best performance, but sometimes the worst. Due to the unstable behavior of the performance for different loops, the FLMS may not be suitable for practical applications. Our TF-TEQ algorithm has good SSNR values for different loops. Although TF-TEQ cannot achieve the best performance for every loop, the steady shortened behavior makes our algorithm suitable for practical applications. Besides, as shown in Section IV, our algorithm has the advantage of low complexity. Thus, our algorithm is more attractive than other TEQ algorithms.

After the verification of our TF-TEQ algorithm, we perform the system simulation of combining TEQ and FEQ. First, we train the TEQ by using one of the three on-line algorithms followed by the training of FEQ. When the coefficients of TEQ are available, we continue sending training symbols into the system. Finally, we estimate the SNR value of each subchannel by computing the difference between the equalized output, $Y(k)$, and the training symbol, $X(k)$. We can consider the SSNR as the performance measurement of TEQ algorithm in time domain. On the contrary, the SNR is the measurement of TEQ performance in frequency domain.

The simulation results of $S N R$ of the eight channels are summarized in Table 4. By using FLMS algorithm, most testing loops have the best $S N R$, but a few loops have the worst. The reason is that these loops cannot be shortened well by using FLMS algorithm. Due to the severe ISI, the $S N R$ cannot be improved efficiently. Hence, the FLMS algorithm is not a good solution for TEQ training. Our TF-TEQ algorithm can achieve better SNR than TLMS for most loops. From the viewpoint of time domain, TLMS seems to achieve better shortening performance than ours. However, our algorithm has the better performance than TLMS in frequency domain. From these results, we conclude that our algorithm is more robust than TLMS and FLMS in the frequency domain.

Table 3 SSNR value of eight testing channels using different algorithms (500 training symbols).

\begin{tabular}{|cc||ccc|}
\hline & & & \\
Channel & Before & FLMS & TLMS & Ours \\
\hline T1.601 Loop\#7 & 20.73 & 25.6 & 42.9 & 41.2 \\
T1.601 Loop\#9 & 19.78 & 35.1 & 38.2 & 39.0 \\
T1.601 Loop\#13 & 19.65 & 25.8 & 41.6 & 41.7 \\
CSA Loop\#4 & 17.51 & 38.3 & 42.0 & 37.2 \\
CSA Loop\#6 & 21.32 & 47.6 & 47.8 & 45.8 \\
CSA Loop\#7 & 19.08 & 34.4 & 46.0 & 37.5 \\
CSA Loop\#8 & 19.41 & 28.1 & 51.9 & 42.2 \\
Mid CSA loop & 22.83 & 45.7 & 45.2 & 42.1 \\
\hline
\end{tabular}

Table 4 SNR Comparison of three on-line TEQ algorithms

\begin{tabular}{|c|c|c|c|c|}
\hline Channel & $\begin{array}{c}S N R \text { before } \\
\text { shorten }\end{array}$ & FLMS & TLMS & Ours \\
\hline T1.601 Loop\#7 & 18.557 & 29.220 & 31.814 & 34.641 \\
\hline T1.601 Loop\#9 & 19.630 & 36.679 & 29.712 & 35.846 \\
\hline T1.601 Loop\#13 & 18.603 & 31.737 & 35.507 & 37.940 \\
\hline CSA Loop\#4 & 24.697 & 44.748 & 40.522 & 39.474 \\
\hline CSA Loop\#6 & 25.124 & 48.972 & 43.282 & 47.467 \\
\hline CSA Loop\#7 & 23.846 & 38.734 & 42.147 & 40.823 \\
\hline CSA Loop\#8 & 20.946 & 36.044 & 45.253 & 43.248 \\
\hline Mid CSA loop & 30.915 & 50.102 & 41.894 & 45.916 \\
\hline
\end{tabular}

\section{Conclusions}

In this paper, we derived a novel on-line algorithm, which is suitable for practical implementations. We have simulated our algorithm on the ADSL testing loops by using MATLAB. The simulation results show that our training algorithm can effectively shorten the channel response. Compared with TLMS and FLMS, our algorithm can achieve the comparable SNR performance. For some testing loops, our algorithm can even achieve the best performance. Due to the low complexity and the better SNR performance, the proposed TF-TEQ algorithm is very attractive to the implementation of DMT-based ADSL systems.

\section{Reference}

[1] P. J. W. Melsa, R. C. Younce, and C. E. Rohrs, "Impulse response shortening for discrete multitone transceivers," IEEE Trans. Commun., vol.44, pp.1662-1672, Dec. 1996.

[2] Lee, J. S. Chow, and J. M. Cioffi, "Performance evaluation of a fast computation algorithm for the DMT in high-speed subscriber loop," IEEE J. Select. Areas Commun., vol. 13, pp. 1560-1570, Dec. 1995.

[3] W. Chiu, W. K. Tsai, T. C. Liau, "Time-domain channel equalizer design using the inverse power method," in Int. Conf. Commun., vol. 2, pp. 973-977, 1999.

[4] J. S. Chow, J. M. Cioffi, and J. A. C. Bingham, "Equalizer training algorithms for multicarrier modulation systems," in Int. Conf. Commun., Geneva, May 1993, pp.761-765.

[5] C. S. Chang, "Low-complexity equalization algorithms for DMT transceiver," Master Thesis, Dep. Of Communication Engineering, NCTU, 1999.

[6] G. Long, F. Ling, and J. Proakis, "The LMS algorithm with delayed coefficient adaption," IEEE Trans. Acoust. Speech, Signal Processing, vol. 37, pp.1397-1405, Sep. 1989

[7] "Network and Customer Installation Interfaces: Asymmetrical Digital Subscriber Line (ADSL) Metallic Interface," ANSI T1.413, 1995. 